\title{
A Merchant Transmission Approach for Uniform-Price Electricity Markets
}

\author{
Philipp Staudt \\ Karlsruhe Institute of Technology \\ philipp.staudt@kit.edu
}

\author{
Shmuel Oren \\ University of California, Berkeley \\ shmuel@berkeley.edu
}

\begin{abstract}
Uniform-price electricity markets as operated in Germany, for instance, rely on a redispatch mechanism after market clearing to ensure the technical feasibility of generation and consumption schedules with regard to grid constraints. This mechanism determines the costs of congestion management and the welfare loss due to the limited transmission capacity. Therefore, the mechanism is suited to incentivize welfare increasing grid expansion. Depending on the distribution of congestion management costs, it can also align stakeholder interests. In this paper, we present an auction mechanism for transmission grid expansion based on the reduction of redispatch expenditures that theoretically leads to a welfare optimal expansion. The mechanism is applied to a case study in Germany. The results show that the developed mechanism supports an improved planning of grid capacity expansion.
\end{abstract}

\section{Introduction}

Transmission grid congestion management is an often considered topic in energy economics. Steven Stoft calls it "one of the toughest problems in electricity market design" ([1], p. 24. While it is generally desirable to place generation capacity as close to the load as possible if ambient conditions are equal at all locations, it might be beneficial from a welfare point of view to place generation capacity where it works most efficiently in case of different ambient conditions. In practice this means that wind turbines are installed in windy areas and PV panels in sunny areas. In this case, a transmission grid needs to be constructed that allows to transmit energy from generation to load centers. In such an environment congestion might occur if the energy that needs to be transmitted surpasses the capacity constraints of the network. This congestion needs to be managed to ensure a balanced grid operation. The chosen mechanism for short-term congestion management depends on the market design
[2]. Markets that employ locational marginal pricing (i.e., nodal pricing) handle congestion at market clearing by considering network constraints explicitly. Uniform-price markets need a specific mechanism to manage congestion as the market is cleared centrally without consideration of the network. This mechanism is called redispatch as it is a deviation from the original economic dispatch [3]. The compensation of generators for the redispatch can be regulated and is then usually cost-based or it can be organized through a market process in which case it is market-based [4]. Zonal pricing is a compromise between nodal and uniform-pricing. Cross-zonal capacity is explicitly considered during the market clearing, while intra-zonal congestion is managed through redispatch.

Systematic long-term congestion occurs if the generation and consumption patterns are not aligned with the transmission network topology. There are multiple ways to reduce this congestion. For instance, generation capacity with low marginal cost can be constructed close to consumption centers. Another option is transmission grid capacity expansion. Transmission grids are complex networks. It is difficult to determine the optimal expansion because this includes the correct anticipation of future generation and consumption patterns as well as the reaction of stakeholders to grid expansion. The problem has a spatial and a temporal component. Spatial, because the exact location of load and generation determine the flows in the network and temporal because the load and the generation from renewable sources vary with time. It is therefore reasonable to allow for some congestion in peak periods [5]. Additionally, grid expansions are time intensive and often unpopular with the local population [6].

Previous attempts of liberalizing the market for transmission grid expansions are focused on markets with locational marginal pricing. The developed mechanisms are based on financial transmission rights that entitle their owners to transmission congestion rents in case of congestion and differing locational 
prices. However, these designs come with a variety of challenges often rooted in the design of nodal pricing markets. In this paper, we present an approach to incentivize a welfare optimal grid expansion in uniform-price electricity markets through cost-based redispatch. While this congestion management design generally entails the risk of strategic behavior such as inc-dec gaming [7], this is not an issue for the proposed mechanism. The auction design is based on the fact that congestion management costs caused by redispatch exactly reflect the possible welfare gain of a grid expansion. It is a first approach to long-term congestion management in Europe. In this contribution we focus on the German market and its congestion management design. This is an important contribution as no other locational signals exist in European uniform-price electricity markets to reduce long-term structural congestion. In this paper we answer the following research questions: (i) What is the economic effect of cost-based redispatch? (ii) How can cost-based redispatch incentivize grid expansion? (iii) What is the effect of a corresponding auction mechanism on the system design?

\section{Related work}

There are two major approaches for incentivizing transmission grid expansion: Long-term financial transmission rights and a regulatory approach [8]. In the following both designs are briefly introduced and related research is presented. Finally, the incentives of market participants in regard to grid expansion and the redispatch mechanism are discussed.

Merchant transmission Merchant transmission approaches are considered by various authors such as [9, 10]. These approaches have the benefit of incentivizing grid expansion based on economic criteria. These are assessed by private parties who then bear the risk for unnecessary expansion. Merchant transmission approaches are based on the allocation of financial transmission rights (FTRs) to the investors of the corresponding transmission line [11]. The owners of these rights receive the transmission congestion rent if congestion occurs in the grid [12]. The authors of [13] perform one of the first studies on incentive design for electricity grid expansion. They expand on their ideas in [14]. They argue that assigning FTRs can correctly incentivize optimal grid expansion under a few assumptions. However, in [9], the authors argue that these assumptions are quite restrictive. Other authors argue that the payments for FTRs do not sufficiently compensate for the investment in transmission capacity $[15,16,17]$. Other authors argue that congestion revenue in the form of transmission congestion rent is poorly designed to incentivize optimal grid expansion because it does not reflect the potential welfare gain of grid expansion [18]. We follow this line of argumentation in this paper and show in Section 3 how redispatch correctly incentivizes grid expansion in regard to welfare increase. Finally, in [8] the authors combine the merchant approach with the regulatory approach to overcome the problem of lacking compensation for grid expansion through transmission rents. This concept is further tested in [19].

Regulated transmission expansion Germany and most European countries regulate the transmission grid and incentivize the expansion through a revenue-cap incentive regulation [20]. A detailed discussion on the German regulation mechanism is provided in [21]. The author points out that the current regulation incentivizes capital intensive solutions over other short-term measures that increase the operating costs. This is due to the fact that the transmission system operators (TSOs) receive guaranteed interest on their equity. Alternative approaches such as battery storage are therefore not considered as proposed by [22]. Other authors point out that TSOs have incentives to inefficiently inflate the need for grid expansion [23]. Furthermore, the regulation leads to problems when it comes to cross-border transmission expansion projects and the corresponding compensation. In [24], the authors propose a procedure to allocate gains from such projects to the involved parties.

Incentives for transmission grid expansion Besides the financial incentives for grid expansion, it is also important to consider the interests of different market actors. Even if a certain transmission expansion increases the social welfare, it might have adverse effects for some parties. This is especially true for markets with nodal prices, as transmission grid expansion might increase the prices at certain nodes. In [25], the authors consider incentives of different market parties for a certain transmission expansion. They find that the ability to exercise market power can lead to inverted incentives. Furthermore, in nodal pricing markets, grid expansions have an impact on generation investment decisions as they lead to a differing set of prices. This phenomenon is considered in [26]. The authors formulate a multi-stage optimization problem to analyze the strategic responses to transmission expansion. They find several conflicts of interest among market parties. The author of [27] performs an analysis of the welfare effects of transmission grid expansion. 
Among other results, the author points out that a strict unbundling of generation and transmission grid assets is necessary. We further elaborate on this observation later in the paper.

Redispatch Redispatch is the process of adjusting the schedule of producers such that the market result of a uniform-price electricity market becomes feasible with regard to the network constraints [3]. In its ideal form, the theoretical nodal pricing market result is achieved [28]. In Germany, the cost for congestion management through the redispatch mechanism have been increasing in the last few years and reached a record high of 1.4 billion in 2017 after not surpassing 100 million until 2011 [29]. They are expected to reach 4 billion by 2023 [30]. This is caused by the development of renewable generation and the nuclear phase-out [31]. The redispatch mechanism in Germany is cost-based: A power plant is compensated with an amount that is calculated based on a fixed procedure [32] that should represent the operating and market opportunity costs of the power plant. However, the European Commission recently instructed all member states to employ a market-based redispatch mechanism [33]. This is highly debated [34] as such a design might allow for similar strategic behavior as the inc-dec gaming in the Californian market [7].

\section{Cost-based redispatch}

The cost-based redispatch mechanism pays a compensation to power plants that are asked to increase their generation based on their production costs. Power plants in a generation pocket that need to decrease their generation on the other hand, are required to reimburse the TSOs with their marginal cost of generation. Note that they are not required to pay back the uniform market clearing price they received. The difference is socialized and being paid through grid tariffs by the consumers. This means that the producer surplus that is lost due to grid constraints is being covered by consumers. The cost of increasing generation in load pockets is also covered by consumers [3]. The economic consequences are depicted in Fig. 1. The increasing line represents the marginal generation costs at a certain node. The decreasing line represents the saved marginal generation costs through supply from the transmission grid. If there is no congestion, the market clearing price is represented by the intersection of the two curves. The dashed line shows the level of the market clearing price. Congestion is represented by the vertical line. The quantity to the left of the line on the $\mathrm{x}$-axis is the supply from the grid. The quantity between the vertical line and the equilibrium point represents the necessary redispatch. The resulting triangle is the potential welfare gain of a grid expansion. In theory, the potential additional consumer surplus is the area in the triangle above the dashed line and the additional producer surplus the area below the dashed line. However, as stated, the entire costs are borne by the consumers and a grid expansion therefore only affects the consumer surplus. It is important to note that the redispatch cost exactly represents the welfare gain of a non-congested transmission system compared to the status quo. The checkered area below the marginal production cost curve is the amount that generators need to reimburse to the TSOs as this represents the saved operating costs. The entire grey area is the cost for the increased redispatch generation.

The mechanism can be formulated as a two stage optimization problem. First, the market is cleared uniformly. This means that the production cost is minimized without consideration of the transmission grid constraints. For simplicity we ignore ramping constraints and idle times, which are common assumptions [23, 35]. The optimization problem is given in Equ. 1. It considers generation constraints and the balance constraint between generation and demand over all buses and generators.

$$
\begin{array}{ll}
\min & \sum_{t=1}^{T} \sum_{i=1}^{N_{b}} \sum_{j=1}^{J} p_{i, j} \cdot q_{i, j, t} \\
\text { s.t. } & \sum_{i=1}^{N_{b}} \sum_{j=1}^{J} q_{i, j, t}=\sum_{i=1}^{N_{b}} d_{i, t}, \forall t \in T \\
& q_{i, j, t} \leq c_{i, j, t}, \forall i \in N_{b}, \forall j \in J, \forall t \in T \\
& q_{(i, j, t)} \geq 0,, \forall i \in N_{b}, \forall j \in J, \forall t \in T
\end{array}
$$

$\begin{array}{ll}p_{i, j} & \text { Marginal cost of unit } j \text { at node } i \\ q_{i, j, t} & \text { Generation at node } i \text { of unit } j \text { at time } t \\ c_{i, j, t} & \begin{array}{l}\text { Capacity of production unit } j \text { at node } i \\ \text { (at time } t \text { for renewables) }\end{array} \\ d_{i, t} & \text { Demand at node } i \text { at time } t \\ N_{b} & \text { Number of buses (nodes) }\end{array}$

In the second stage after the market clearing, the market result is adjusted to ensure a physically feasible solution. Therefore, the transmission constraints are included as shown in Equ. 2 and redispatch is performed. The formulation is based on a direct current approximation and ignores losses [36]. In the 


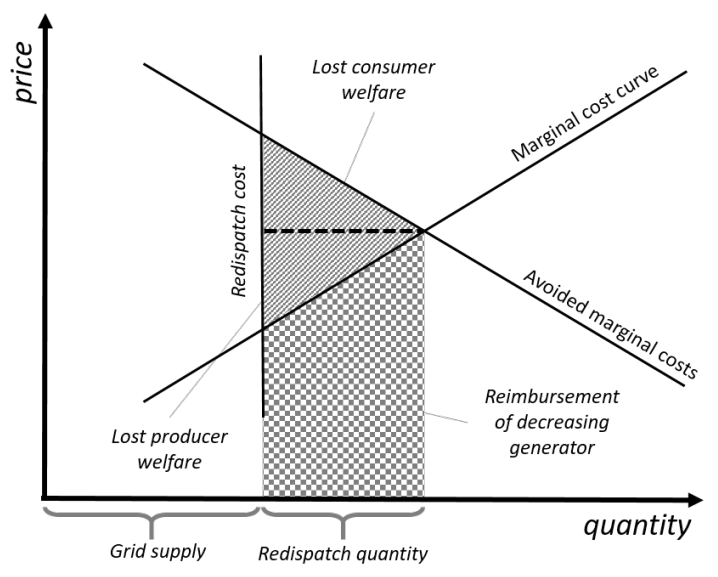

Figure 1. Economics of cost-based redispatch

objective function the marginal cost of production that is reimbursed to the TSOs is subtracted from the cost of increasing generation. The sum of redispatch needs to equal zero and the capacity constraints need to be respected after the redispatch. We use this formulation in the following section to describe the expansion policy.

$$
\begin{array}{ll}
\min & \sum_{t=1}^{T} \sum_{i=1}^{N_{b}} \sum_{j=1}^{J} p_{i, j} \cdot q_{i, j, t}^{\Delta} \\
\text { s.t. } \quad & \sum_{i=1}^{N_{b}} \sum_{j=1}^{J} q_{i, j, t}^{\Delta}=0, \forall t \in T \\
& q_{i, j, t}+q_{i, j, t}^{\Delta} \leq c_{i, j, t}, \forall i \in N_{b}, \forall j \in J, \forall t \in T \\
& q_{(i, j, t)}+q_{i, j, t}^{\Delta} \geq 0, \forall i \in N_{b}, \forall j \in J, \forall t \in T \\
& \left.\mid \sum_{i=1}^{N_{b}-1} H_{(l, i)} \cdot\left(\sum_{j=1}^{J}\left(q_{i, j, t}+q_{i, j, t}^{\Delta}\right)-d_{i, t}\right)\right) \mid \\
& \leq \tau_{l}, \forall t \in T, \forall l \in N_{l}
\end{array}
$$

$q_{i, j, t}^{\Delta} \quad$ Redispatch at node $i$ of unit $j$ at time $t$

$H \quad$ Matrix of power distribution factors

$\tau_{l} \quad$ Transmission capacity of line $l$

$N_{l} \quad$ Number of lines

\section{Transmission grid expansion mechanism}

The economic characteristics of the cost-based redispatch make it a well designed indicator for the economic value of grid expansions. While it has other weaknesses, it reflects the possible welfare gain of additional grid capacity. Based on this design we propose a mechanism that incentivizes a welfare optimal grid expansion. The proposed mechanism operates in three stages: The discovery stage, the auction stage and the compensation stage. All stages are described in the following.

Discovery First, a new transmission expansion project needs to be identified. This is not necessarily the role of the regulator. In the proposed mechanism, any investor has an incentive of proposing a transmission expansion project that she considers beneficial from a social welfare perspective. This project is proposed to the regulator. Several projects might be proposed to the regulator at the same time. It is important to establish a decision rule for the regulator to prioritize projects because they might affect each other and one project might not be profitable anymore if another one is realized first. An approach to prioritize grid expansion projects is proposed in [37]. Once the regulator has chosen the next project, it is made public. Any interested party can evaluate the project and later participate in the auction stage. At this point, it is important that the regulator defines the generation portfolio that is later considered in the compensation stage.

Auction Once the project is evaluated and the assumed generation portfolio is established, investors can bid on the project. Their bid consists of the time period, during which they want to receive the reduction in redispatch costs (and thus the welfare gain) that is achieved through the proposed transmission expansion project. The mechanism is implemented as a Vickrey auction to ensure incentive compatibility. This auction is similar to tendering auctions such as mobile frequencies and can learn from the experiences in these processes [38]. The investor who bids the shortest compensation period wins the auction and receives the compensation for the time period bid by the runner up investor.

Compensation Finally, once the project is developed, the investors are compensated. In each market clearing period, the need for redispatch is calculated following a transparent and previously introduced process. This is done using the former grid topology and the grid topology including the expanded lines. The difference in redispatch costs is paid to the investors. As grid expansion may have a negative effect on the overall grid capacity, this amount can be negative, in which case the investors pay to the TSOs. The redispatch costs are calculated using the assumed generation portfolio that 
the regulator announced in the Discovery and any new generation capacity addition. The importance of this step is further discussed in Section 6. The payments to investors are socialized to the consumers who would have had to pay redispatch costs in the same amount if the grid expansion project had not been realized.

\subsection{Mechanism characteristics}

The cost-based redispatch mechanism itself has certain flaws. For example, it causes additional cost and it only discovers the welfare optimal economic dispatch if TSOs optimize their grids correctly. Additionally, it offers the possibility of gaming the market through a variation of the inc-dec gaming [32]. However, with regards to incentivizing grid expansion it has favorable properties which are discussed in the following.

1. Consumers do not pay more than without the mechanism.

Without the mechanism it would be necessary to use redispatch to ensure a balanced transmission system. These costs would be covered by the consumers. Therefore, payments for an addition to the network do not create additional costs for consumers. However, once the compensation period is over, the consumers benefit from reduced congestion management costs. Therefore, the approach can be characterized as a form of a split the savings strategy that has already been formulated in [39] for electricity pool markets.

\section{Incentives of stakeholders are aligned.}

As the congestion management costs are socialized, the consumers are equally interested in a welfare increasing expansion of the transmission system. Therefore, it is easier to allocate costs, as rules like the beneficiary pays that is formulated in FERC order No. 1000 are not necessary. This does of course not address the "not-in-my-backyard phenomenon" [6] but from a financial viewpoint all consumers are on the same side. The expansion has no impact on the wholesale electricity price for consumers. Furthermore, as the wholesale market is not impacted by transmission grid expansions, the generators should be agnostic to grid expansion.

\section{The compensation covers the investment.}

As the transmission expansions are compensated by the welfare gain they create, the compensation covers the investment. If this is not the case, then the investment is inefficient to begin with. It is of course possible that an investor wrongly anticipates future generation and load patterns. However, in an ideal world with perfect foresight, the mechanism leads to a a welfare optimal grid expansion.

4. The risk of inefficient expansion is borne by investors.

This advantage is related to the previous discussion. It is still possible that the grid is expanded inefficiently if an investor makes mistakes in her forecasts of future generation and consumption patterns. While under the regulatory approach this risk is borne by the consumers who compensate TSOs for any grid expansion regardless of its efficiency, it is now transferred to private investors who act on the (un)certainty of their own forecasts.

5. The market finds the welfare optimal solution by itself.

Finally, the mechanism does not require regulatory oversight of the grid expansion and development. The market actors have incentives of proposing welfare efficient projects. There are no mixed incentives as a degradation of the grid causes payback to the TSOs. The approach therefore allows for a liberalization of the transmission grid expansion market.

\subsection{Investment optimization}

An investor needs to consider the future generation and consumption patterns in order to determine an optimal grid expansion with regard to the possible payback. She therefore needs to solve the uniform market clearing problem followed by optimizing the grid expansion considering expansion and redispatch costs. It is important to note that transmission lines often need to be expanded in joint projects rather than line by line. An individual expansion of a line might only move congestion from one line to the next which does not necessarily decrease congestion costs but might even increase them if more expensive power plants are needed to cure the new congestion. The optimization problem is given in Equ. 3. It is an extension of the redispatch mechanism given in Equ. 2. In its given formulation, line capacity can both be expanded or newly developed. 


$$
\begin{array}{ll}
\min \quad & \sum_{t=1}^{T} \sum_{i=1}^{N_{b}} \sum_{j=1}^{J} p_{i, j} \cdot q_{i, j, t}^{\Delta}+\sum_{l=1}^{L} \tau_{l}^{e x p} \cdot p_{l}^{\exp } \\
\text { s.t. } \quad & \sum_{i=1}^{N_{b}} \sum_{j=1}^{J} q_{i, j, t}^{\Delta}=0, \forall t \in T \\
& q_{i, j, t}+q_{i, j, t}^{\Delta} \leq c_{i, j, t}, \forall i \in N_{b}, \forall j \in J, \forall t \in T \\
& q_{(i, j, t)}+q_{i, j, t}^{\Delta} \geq 0, \forall i \in N_{b}, \forall j \in J, \forall t \in T \\
& \left.\mid \sum_{i=1}^{N_{b}-1} H_{(l, i)} \cdot\left(\sum_{j=1}^{J}\left(q_{i, j, t}+q_{i, j, t}^{\Delta}\right)-d_{i, t}\right)\right) \mid \\
& \leq\left(\tau_{l}+\tau_{l}^{e x p}\right), \forall t \in T, \forall l \in N_{l}
\end{array}
$$

$\begin{array}{ll}\tau_{l}^{\text {exp }} & \text { Expansion of line } l \\ p_{l}^{\text {exp }} & \text { Cost of expansion of line } l \text { per MW and period }\end{array}$

\section{Analytical example and case study}

Consider the system given in Fig. 2. The cheaper production is located at node A with marginal cost of production $m c_{i}$ and the generation $q_{i}$. The load $l_{i}$ is higher at note $\mathrm{B}$ with $i \in\{A, B\}$. Using nodal pricing, the price at node $\mathrm{A} p_{A}$ is $9 \$ / \mathrm{MWh}$ and $p_{B}$ is 12 $\$ / \mathrm{MWh}$. This results in total payments of the consumers of $165 \$$, a producer surplus of $76.5 \$$ and a congestion rent of $12 \$$. Note that an expansion of the line to a capacity $t_{A B}$ to $5 \mathrm{MW}$ would not be in the interest of the holder of financial transmission rights for that line because it would eliminate the congestion and therefore the congestion rent. Now, we consider a uniform-price market with cost-based redispatch. The market clears without consideration of the transmission constraint. Therefore, the market clears at $p_{A}=p_{B}=10 \$ / \mathrm{MWh}$, $q_{A}=10 \mathrm{MWh}$ and $q_{B}=5 \mathrm{MWh}$. This schedule is not feasible as the necessary transmission from node $\mathrm{A}$ to $\mathrm{B}$ is $5 \mathrm{MW}$, which exceeds the line capacity. Therefore, the generation at node $\mathrm{A}$ is reduced by $1 \mathrm{MW}$ and the generation at node B is increased by $1 \mathrm{MW}$. At node A the generator reimburses the system operator for the avoided cost of production of $9.5 \$$. The generator at node $B$ receives $11 \$$ for the increase in generation. Therefore, the cost for redispatch is $1.5 \$$. Now, we assume an investor that is willing to expand the line and that the parameters of the system never change. The costs of expanding the line capacity by $1 \mathrm{MW}$ is $c_{A B}$ per considered period. In practice that period can be an hour or 15 minutes, depending on how often the market

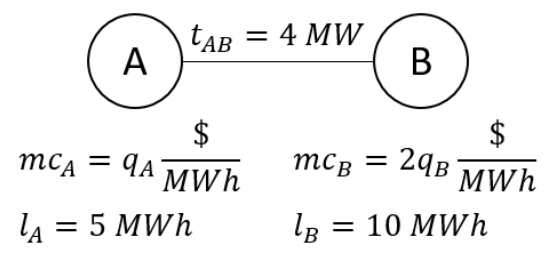

Figure 2. Two-node transmission system

is cleared and redispatch is performed. However, given the assumptions of power and energy in this example it is by hour. Therefore, if $c_{A B}>1.5 \$$ it is not reasonable to expand the line. In that case the cost of line expansion would exceed its benefit and the investor would not consider it. Note, that the interest of the investor and the system welfare considerations are aligned: If congestion is avoided in the system, the consumers pay a total of $150 \$$, the producer surplus is reduced by $1.5 \$$ and the transmission rent of $12 \$$ is avoided. Therefore, the total welfare increase of an uncongested system is 1.5 $\$$. This way, the correct market signals are sent to the investor and welfare optimal transmission expansion is performed.

\subsection{Simulative evaluation}

In order to assess the effects of the mechanism, it is evaluated using an abstracted version of the German electricity transmission grid. To do so, Germany is divided into a zonal model with 5 bidding zones. The setup is given in Fig. 3. It loosely corresponds to the regions of the four German TSOs. Only the central TSO TenneT is divided into two zones to account for the North-South division of the German electricity system: Lignite and wind generation is mostly located in the North and North-East while most consumption occurs in the South. The transmission capacity is based on the static grid models of the TSOs TenneT [40], Amprion [41], TransnetBW [42] and 50Hertz [43]. In the following simulation each zone corresponds to one node. This way we can optimize the load flow as if we had a full network model. We use empirical data for renewable generation and load from [44]. The conventional generation capacity is based on the capacity reported by the German regulator [45]. The technology specific marginal costs of generation are based on [46]. We assume a static optimization. That means that the investor does not foresee the development of generation and demand patterns but always optimizes the generation expansion based on the current patterns. This is done here to illustrate the effects of the mechanism. We only consider line reinforcement and no construction of additional lines 


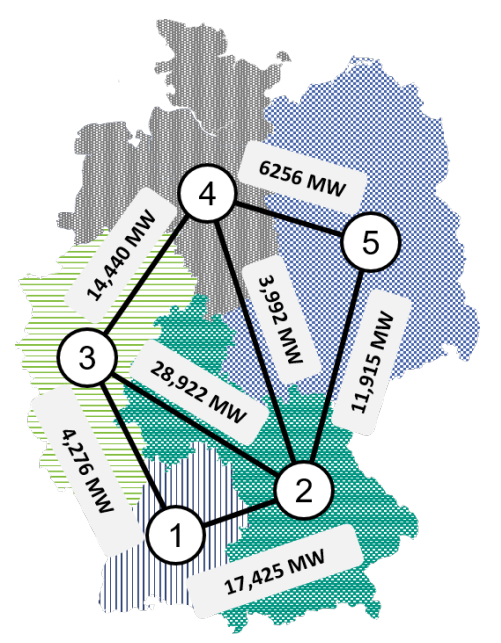

Figure 3. Zonal model of German transmission grid

as this easily becomes a very complex problem [2]. A similar approach is used in [47]. The authors only allow for one additional line along the North-South division in Germany. Such a line is already included in our model and therefore does not need to be considered explicitly. We assume all lines to be of equal length and only distinguish between different line capacities. Similarly, [23] only consider line length between zone centers in a zonal representation of the German system. We base the cost per MW of capacity on the HVDC project SuedOstLink. This model calculation serves as an illustration of the mechanism. In future research we will include more detailed transmission network data for the German system including a forecast of the development of spatial and temporal generation and consumption patterns.

Static results The simulation results for the year 2016 are given in Table 1. The numbers show that the expansion using the redispatch incentive mechanism leads to the same welfare optimal expansion as under a nodal pricing market design. Through this expansion, the redispatch costs are reduced from 95 million to 39 million Euro per year. It is considerable that $41 \%$ of the total redispatch in the base scenario should be managed in the short-term through congestion management rather than through grid expansion. It underlines the paradigm that the grid should not be expanded to transmit the last $k W h$ [5]. However, this is incentivized through the German incentive regulation [48]. Expanding the grid such that no congestion occurs anymore increases the overall yearly costs by 700 million Euro and is by far the most expensive option. Even if reducing congestion is a priority objective over economic considerations, the reduction of redispatch to $10 \%$ of its original value

\begin{tabular}{lrrrr} 
Scenario & $\begin{array}{c}\text { Consumer } \\
\text { costs }\end{array}$ & $\begin{array}{c}\text { Producer } \\
\text { surplus }\end{array}$ & $\begin{array}{c}\text { Cong. rent/ } \\
\text { redispatch }\end{array}$ & $\begin{array}{c}\text { Grid exp. } \\
\text { (in MW) }\end{array}$ \\
\hline Nodal pricing & 9,453 & 4,305 & 164 & 592 \\
Redispatch optimal & 9,311 & 4,289 & 39 & 592 \\
Redispatch 90\% & 9,331 & 4,289 & 9 & 1,401 \\
No congestion & 10,035 & 4,289 & 0 & 12,806 \\
No expansion & 9,329 & 4,289 & 95 & 0 \\
\hline
\end{tabular}

Table 1. Welfare evaluation of different scenarios in million Euro

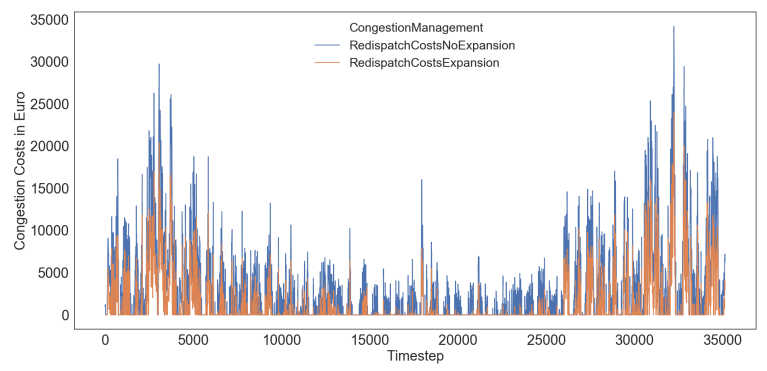

Figure 4. Congestion management costs over the course of 2016

is only 21 million Euro more expensive per year than the optimal solution. Note that both options are not economically reasonable and would not be undertaken by an investor under the proposed auction mechanism. Furthermore, the results show that generators should be agnostic to line expansions as their producer surplus does not change in a market with uniform-price design. In Fig. 4 the need for redispatch in the course of 2016 is displayed in a 15-minute resolution. It shows that the need for redispatch is especially high during the winter month which corresponds to the actual redispatch quantities in Germany. It can also be seen that high spikes of redispatch persist even with the grid expansion. This is caused by the intermittency of renewable generation that makes it even more important to consider short-term congestion management as a viable alternative to long-term grid expansion.

Sequential evaluation In order to assess the effects of an evaluation over multiple years, the mechanism is applied to the years 2016 to 2018. Additionally, the year 2018 is considered a second time as another data point with a finalized nuclear phase-out that will be completed in Germany in 2022. As most of the still operational nuclear power units are situated in the South, this further increases the imbalance between the North and the South [49]. Each year is again statically considered and the payments for an expansion based on that year are calculated in the next meaning that an instantaneous project realization is assumed. The approach allows a perspective on the load and generation uncertainty 


\begin{tabular}{lrrrr} 
Year & $\begin{array}{r}\text { Red. } \\
\text { costs }\end{array}$ & $\begin{array}{c}\text { Red. costs } \\
\text { w/o exp. }\end{array}$ & $\begin{array}{c}\text { Opt. exp. } \\
\text { (MW) }\end{array}$ & $\begin{array}{r}\text { Payment } \\
\text { investors }\end{array}$ \\
\hline 2016 & 95 & 95 & 592 & 0 \\
2017 & 136 & 187 & 909 & 52 \\
2018 & 126 & 271 & 858 & 145 \\
2018 (w/o Nuc.) & 76 & 317 & 429 & 241 \\
\hline
\end{tabular}

Table 2. Grid development and associated costs in million Euro over four year period

over several years: The expansion is always realized ex-post based on the trend in the respective year and the compensation is then evaluated on the following year. Therefore, the uncertain infeed of intermittent renewable capacity and load can have an impact on the investor's revenue. It can be seen that even though this is a naïve strategy, it yields positive profits. This shows that the agglomeration of cheap generation capacity in the North is continuing. The shown expanded capacity is always a reinforcement of the line between TenneT North zone 4 and TenneT South zone 2. No other lines need expansion. In every year, the reinforcement of that line is increased. It can be seen from the last column that the theoretical redispatch in the last year is reduced by roughly $75 \%$ through the mechanism which is equivalent to 241 million Euro. This amount is reimbursed to the investor.

Overall, we find that the mechanism incentivizes a grid expansion that is welfare optimal and that takes into account that certain congestion management should occur through other measures than grid expansion. This leaves avenues for further research which are discussed in the following section along with practical considerations for the mechanism.

\section{Discussion}

The proposed mechanism has a few drawbacks that need to be considered.

Redispatch procedure First, the redispatch procedure needs to be clearly defined and all associated calculations need to be agreed upon before the auction begins. This includes the calculation of the virtual marginal cost of production of redispatched units and the exact optimization problem formulation of the redispatch selection procedure. The calculation of marginal costs of production needs to include a possible extension to newly constructed units. It needs to be considered how temporary unavailability is treated as well. These aspects are essential and need to be carefully designed as they form the basis for the optimal grid expansion.
Forecasts and risk Transmission lines are written off over 40 years [23]. Even if this would no longer be the case under the proposed auction mechanism as investors only need to consider their bid time horizon, the amortization period might still be long. Investment decisions for such a time horizon need to be carefully considered and involve a lot of uncertainty. It is unclear how this uncertainty impacts the decisions for grid expansion. If it is prohibitively high, then necessary grid expansions are not considered or only few investors participate in the bidding. This would decrease the efficiency of the mechanism. However, it is debatable whether the public should finance such projects if private investors consider the related risk as too high. Furthermore, if forecasts are far off, the mechanism would result in an inefficient grid expansion that would increase public opposition against any transmission grid expansion projects and damage the efficiency of the approach in the long run.

Competition One central element of efficient markets and auctions is competition [38]. Therefore, it is important to attract sufficient competitors for each project. The actors and investors for such expansion projects are currently unknown as such projects have not yet been tendered. It is however not difficult to test the design for a few smaller projects before deploying it for all grid expansions. This would help to judge the landscape of potential participants.

Unbundling For the mechanism to work it is important to ensure a strict unbundling between generation and transmission. This should be the case in most countries but needs to be strictly overseen [50]. A generation company could otherwise give notice to a certain investor of a planned expansion of regional cheap electricity generation capacity that could potentially change the nature of congestion. An investor could now act on this knowledge and propose an according grid expansion that would not be profitable without the generation expansion. The investor would therefore have an unfair competitive advantage. Furthermore, generation companies could bid into the market in a way to increase the virtual redispatch on a newly constructed line to increase the revenue of the investor. Such strategic considerations are to be avoided through a rigorous regulation on collusive behavior.

Decommissioning power plants Finally, one important aspect is the change in virtual redispatch costs if certain power plants are decommissioned. The virtual redispatch that would occur without newly constructed lines. Usually, the regulator in Germany 
would prevent generators from decommissioning if the power plant is important for the system stability. However, due to new transmission capacity, a power plant could no longer be needed. If such a power plant is shut down, it is possible that virtual redispatch could no longer be performed because no other regional generation capacity exists or the costs could become prohibitively high. Therefore, the regulator needs to establish a base case of available generation capacity when a project is auctioned off. This generation capacity is then later assumed to be available even if specific plants are decommissioned. However, new generation technology is to be included in the calculation of redispatch.

\section{Conclusion}

In this paper we propose an auction mechanism that incentivizes transmission grid expansion based on cost-based redispatch that is used in many European countries with a uniform-price electricity market to achieve feasible results with regard to grid constraints. We provide an economic description of the cost-based redispatch mechanism and show that the redispatch costs correspond to the welfare gain of a line expansion. Furthermore, we describe how this mechanism aligns financial interests of stakeholders in the electricity sector. We then provide an analytical illustrative example and then apply the mechanism to an abstracted zonal version of the German transmission grid. The evaluation shows that the mechanism leads to the same welfare optimal grid expansion as nodal pricing. This is due to the fact that the two optimization problems aim to find the same solution. Furthermore, it is shown that the optimal solution is considerably cheaper than an expansion to transmit the last generated kWh. Additionally, the results show that generators are theoretically agnostic to a grid expansion. The mechanism is then applied over a four year period to illustrate its dynamics. We find that the development of generation and consumption patterns in Germany point in the same direction over the years such that a static optimization on individual years would currently be profitable for an investor. Finally, possible limitations of the proposed auction mechanism are discussed and addressed. With that we answer the considered research questions: (i) Cost-based redispatch exactly represents the possible welfare gain of expanding the transmission system to a state without congestion. (ii) Using this characteristic, transmission expansion can be incentivized through redispatch costs because a transmission line is beneficial if and only if it reduces redispatch costs beyond the investment costs for its construction. (iii) Therefore, an auction mechanism that pays compensation based on reduced redispatch costs leads to a welfare optimal grid expansion.

The objective of this paper is the introduction and illustration of the auction mechanism for the expansion of transmission capacity. The application to the zonal version of the German transmission grid limits the generalizability of the results to the entire transmission system. Furthermore, rather than considering static choices, a projection of the expansion of regional generation capacity should be considered. Finally, the mechanism should be experimentally evaluated to be able to judge the behavior of participants. These extensions will be addressed in future research.

\section{References}

[1] S. Stoft, "Power system economics," Journal of Energy Literature, vol. 8, pp. 94-99, 2002.

[2] M. Weibelzahl, "Nodal, zonal, or uniform electricity pricing: how to deal with network congestion," Frontiers in Energy, vol. 11, no. 2, pp. 210-232, 2017.

[3] A. Nüßler, Congestion and redispatch in Germany. A model-based analysis of the development of redispatch. $\mathrm{PhD}$ thesis, Universität zu Köln, 2012.

[4] L. Hirth and S. Glismann, "Congestion management: From physics to regulatory instruments," 2018.

[5] S. Stoft and F. Lévêque, "Competitive electricity markets and sustainability," Competitive Electricity Markets and Sustainability, 2006.

[6] P. Devine-Wright, "Explaining NIMBY objections to a power line: The role of personal, place attachment and project-related factors," Environment and behavior, vol. 45, no. 6, pp. 761-781, 2013.

[7] Z. Alaywan, T. Wu, and A. D. Papalexopoulos, "Transitioning the California market from a zonal to a nodal framework: An operational perspective," in IEEE PES Power Systems Conference and Exposition, 2004. , pp. 862-867, IEEE, 2004.

[8] W. Hogan, J. Rosellón, and I. Vogelsang, "Toward a combined merchant-regulatory mechanism for electricity transmission expansion," Journal of regulatory Economics, vol. 38, no. 2, pp. 113-143, 2010.

[9] P. Joskow and J. Tirole, "Merchant transmission investment," The Journal of industrial economics, vol. 53, no. 2, pp. 233-264, 2005.

[10] G. Brunekreeft, "Regulatory issues in merchant transmission investment," Utilities Policy, vol. 13, no. 2, pp. 175-186, 2005.

[11] T. Kristiansen and J. Rosellon, "Merchant electricity, transmission expansion: A European case study," Energy, vol. 35, no. 10, pp. 4107-4115, 2010.

[12] W. W. Hogan, "Contract networks for electric power transmission," Journal of regulatory economics, vol. 4, no. 3, pp. 211-242, 1992.

[13] J. B. Bushnell and S. E. Stoft, "Electric grid investment under a contract network regime," Journal of Regulatory Economics, vol. 10, no. 1, pp. 61-79, 1996. 
[14] J. B. Bushnell and S. E. Stoft, "Improving private incentives for electric grid investment," Resource and Energy Economics, vol. 19, no. 1-2, pp. 85-108, 1997.

[15] L. Cameron, "Transmission investment: Obstacles to a market approach," The Electricity Journal, vol. 14, no. 2, pp. 25-38, 2001

[16] G. Brunekreeft, K. Neuhoff, and D. Newbery, "Electricity transmission: An overview of the current debate," Utilities Policy, vol. 13, no. 2, pp. 73-93, 2005.

[17] F. J. Rubio-Odériz and I. J. Perez-Arriaga, "Marginal pricing of transmission services: A comparative analysis of network cost allocation methods," IEEE Transactions on Power systems, vol. 15, no. 1, pp. 448-454, 2000.

[18] M. Barmack, P. Griffes, E. Kahn, and S. Oren, "Performance incentives for transmission," The Electricity Journal, vol. 16, no. 3, pp. 9-22, 2003.

[19] W.-P. Schill, J. Egerer, and J. Rosellón, "Testing regulatory regimes for power transmission expansion with fluctuating demand and wind generation," Journal of Regulatory Economics, vol. 47, no. 1, pp. 1-28, 2015.

[20] G. Brunekreeft and D. Bauknecht, "Energy policy and investment in the German power market," Electricity Market Reform: An International Perspective, Elsevier, pp. 235-264, 2006.

[21] P. Matschoss, B. Bayer, H. Thomas, and A. Marian, "The German incentive regulation and its practical impact on the grid integration of renewable energy systems," Renewable Energy, vol. 134, pp. 727-738, 2019.

[22] M. Weibelzahl and A. Märtz, "Optimal storage and transmission investments in a bilevel electricity market model," Annals of Operations Research, pp. 1-30, 2017.

[23] C. Kemfert, F. Kunz, and J. Rosellón, "A welfare analysis of electricity transmission planning in Germany," Energy Policy, vol. 94, pp. 446-452, 2016.

[24] M. Kristiansen, F. D. Munoz, S. Oren, and M. Korpås, "Efficient Allocation of Monetary and Environmental Benefits in Multinational Transmission Projects: North Sea Offshore Grid Case Study," in Heading Towards Sustainable Energy Systems: Evolution or Revolution?, 15th IAEE European Conference, Sept 3-6, 2017, International Association for Energy Economics, 2017.

[25] E. E. Sauma and S. S. Oren, "Do generation firms in restructured electricity markets have incentives to support social-welfare-improving transmission investments?," Energy Economics, vol. 31, no. 5, pp. 676-689, 2009.

[26] E. E. Sauma and S. S. Oren, "Economic criteria for planning transmission investment in restructured electricity markets," IEEE Transactions on Power Systems, vol. 22, no. 4, pp. 1394-1405, 2007.

[27] T.-O. Léautier, "Transmission constraints and imperfect markets for power," Journal of Regulatory Economics, vol. 19 , no. 1, pp. 27-54, 2001.

[28] C. Maurer, C. Zimmer, and L. Hirth, "Bericht fr das Bundesministerium fr Wirtschaft und Energie: Nodale und zonale Strompreissysteme im Vergleich,” 2018.

[29] B. Bundesnetzagentur, "Monitoringbericht 2018," 2018.

[30] D. Bundesregierung, "Schriftliche Frage an die Bundesregierung durch Oliver Krischer," 2016.
[31] K. Trepper, M. Bucksteeg, and C. Weber, "Market splitting in Germany-New evidence from a three-stage numerical model of Europe," Energy Policy, vol. 87, pp. 199-215, 2015.

[32] P. Staudt, Y. Traeris, B. Rausch, and C. Weinhardt, "Predicting Redispatch in the German Electricity Market using Information Systems based on Machine Learning," 2018.

[33] European Commission, "Clean energy for all Europeans," COM (2016), vol. 860, 2016.

[34] L. Hirth and I. Schlecht, "Market-Based Redispatch in Zonal Electricity Markets," 2018.

[35] V. Grimm, A. Martin, C. Sölch, M. Weibelzahl, and G. Zöttl, "Market-Based Redispatch May Result in Inefficient Dispatch," 2018.

[36] F. Li and R. Bo, "DCOPF-based LMP simulation: algorithm, comparison with ACOPF, and sensitivity," IEEE Transactions on Power Systems, vol. 22, no. 4, pp. 1475-1485, 2007.

[37] M. Franken, A. B. Schrief, H. Barrios, and A. Schnettler, "Network reinforcement applying redispatch-based indicators," in 2018 53rd International Universities Power Engineering Conference (UPEC), pp. 1-6, IEEE, 2018.

[38] P. Klemperer, "How (not) to run auctions: The european $3 \mathrm{~g}$ telecom auctions," European Economic Review, vol. 46, no. 4-5, pp. 829-845, 2002.

[39] K. Doty and P. McEntire, "An analysis of electric power brokerage systems," IEEE Transactions on Power Apparatus and Systems, no. 2, pp. 389-396, 1982.

[40] TenneT, "Static network model," 2017.

[41] Amprion, "Static network model," 2017.

[42] TransnetBW, "Static network model," 2017.

[43] 50Hertz, "Static network model," 2017.

[44] Bundesnetzagentur, "SMARD Strommarktdaten," 2019.

[45] Bundesnetzagentur, "Kraftwerksliste," 2017.

[46] F. Leuthold, H. Weigt, and C. Von Hirschhausen, "Efficient pricing for European electricity networks-The theory of nodal pricing applied to feeding-in wind in Germany," Utilities Policy, vol. 16, no. 4, pp. 284-291, 2008 .

[47] D. Gunkel and D. Möst, "The German transmission grid expansion in long-term perspectiveWhat is the impact of renewable integration?," in 11th International Conference on the European Energy Market (EEM14), pp. 1-6, IEEE, 2014.

[48] A. Weber, T. Beckers, P. Behr, N. Bieschke, S. Fehner, and C. von Hirschhausen, "Longterm power system planning in the context of changing policy objectives-conceptual issues and selected evidence from europe," Studie im Auftrag der Smart Energy for Europe Platform (SEFEP), 2013.

[49] K. Bruninx, D. Madzharov, E. Delarue, and W. D'haeseleer, "Impact of the German nuclear phase-out on Europe's electricity generationA comprehensive study," Energy Policy, vol. 60, pp. 251-261, 2013.

[50] M. Chawla and M. G. Pollitt, "Global trends in electricity transmission system operation: where does the future lie?," The Electricity Journal, vol. 26, no. 5, pp. 65-71, 2013. 